\title{
Convergence of Variational Iteration Method for Fractional Delay Integrodifferential-Algebraic Equations
}

\author{
Yayun Fu, Hongliang Liu, and Aiguo Xiao \\ School of Mathematics and Computational Science, Hunan Key Laboratory for Computation and Simulation in \\ Science and Engineering, Xiangtan University, Xiangtan, Hunan 411105, China
}

Correspondence should be addressed to Hongliang Liu; lhl@xtu.edu.cn

Received 19 September 2016; Accepted 22 November 2016; Published 16 May 2017

Academic Editor: Jean J. Loiseau

Copyright (C) 2017 Yayun Fu et al. This is an open access article distributed under the Creative Commons Attribution License, which permits unrestricted use, distribution, and reproduction in any medium, provided the original work is properly cited.

Fractional order delay integrodifferential-algebraic equations are often used for many practical modeling problems in science and engineering, which have time lag, memory, constraint limit, and so forth. These yield some difficulties in numerical computation. The iterative methods are good choice. In the present paper, we construct variational iteration method for solving them by using the appropriate restricted variation. This overcomes the difficulties caused by limitations of large storage amount and algebraic constraint and extends the previous conclusions.

\section{Introduction}

Fractional delay integrodifferential-algebraic equations (FDIDAEs) are often used for modeling many science and engineering problems with memory and algebraic constraints, such as flexible multibody dynamics and integrated circuits. Recently, fractional integrodifferential equations (FIDEs) have received much attention; for instance, the stability and asymptotic stability of FIDEs are studied in [1-3]; the numerical methods for solving FIDEs can be found in [47]; for the approximate analytical methods for solving FIDEs, the readers can refer to [8-10]. The studies on differentialalgebraic equations (DAEs) are mainly concentrated in qualitative analysis as well as convergence and stability of numerical methods. For instance, the structural characteristics and asymptotic stability of the (neutral) DAEs are presented in $[11,12]$; the convergence results of one-leg methods, RungeKutta methods, BDF methods, and linear multistep methods for DAEs are obtained in [13-16]; the stability of Runge-Kutta methods and Rosenbrock methods for (neutral) DAEs are studied in $[17,18]$. As for integrodifferential-algebraic equations, only a few studies have been undertaken, for instance, the convergence and stability of Runge-Kutta methods [1921].

The variational iteration method (VIM) is one of the important methods used to obtain approximate analytical solutions [22-25] and possesses some good properties, such as flexibility, convenience, accuracy, and less storage. In particular, this method was used to solve pantograph equations $[26,27]$, differential (integral) equations [28-30], fractional differential (integral) equations [31-34], delay differentialalgebraic equations [35] and fractional differential-algebraic equations [36], and so forth.

As far as we know, there are few works about numerical methods (including the VIM) for FDIDAEs. The aim of this paper is to use the VIM to solve FDIDAEs and obtain the corresponding convergence results.

\section{Convergence}

Consider the initial value problems of FDIDAEs.

$$
\begin{aligned}
& D_{*}^{\alpha} x(t)=f\left(x(t), x(\omega(t)), \int_{t-\tau}^{t} h(x(s), y(s)) d s,\right. \\
& \quad y(t), y(\phi(t))), \quad t \in[0, T], \\
& 0=g(x(t), y(t)), \quad t \in[0, T], \\
& x(t)=\varphi(t), \\
& y(t)=\psi(t),
\end{aligned}
$$


$D_{*}^{\alpha} x(t)$ denotes the Caputo derivative of order $\alpha, m-1<\alpha \leq$ $m, m \in N$, the delay functions $\omega(t)$ and $\phi(t)$ satisfy $\omega(t) \epsilon$ $[-\tau, t], \phi(t) \in[-\tau, t], f: R^{n_{1}} \times R^{n_{1}} \times R^{n_{1}} \times R^{n_{2}} \times R^{n_{2}} \rightarrow R^{n_{1}}$, and $g: R^{n_{1}} \times R^{n_{2}} \rightarrow R^{n_{2}}$ are smooth vector functions on the real Euclidean spaces, and $h: R^{n_{1}} \times R^{n_{2}} \rightarrow R^{n_{1}}$ satisfies the Lipschitz condition

$$
\left\|h\left(v_{1}, \theta_{1}\right)-h\left(v_{2}, \theta_{2}\right)\right\| \leq \gamma_{1}\left\|v_{1}-v_{2}\right\|+\gamma_{2}\left\|\theta_{1}-\theta_{2}\right\|,
$$

where $v_{1}, v_{2} \in R^{n_{1}}, \theta_{1}, \theta_{2} \in R^{n_{2}}$, the $(m-1)$-order derivatives of initial value functions $\varphi:[-\tau, 0] \rightarrow R^{n_{1}}$ and $\psi:[-\tau, 0] \rightarrow$ $R^{n_{2}}$ are continuous, the Jacobian matrix $g_{y}$ is invertible, and $f_{i}^{\prime}$ is bounded $\left(f_{i}, i=1,2, \ldots, 5\right.$, denotes the partial derivatives of the function $f$ to $i$ th variable) in a neighborhood of the exact solution. We assume that system (1) has smooth solutions $x(t), y(t)$. Throughout this article, $\|\cdot\|$ denotes the standard Euclidean norm, and the matrix norm is subordinate to $\|\cdot\|$.

Applying the VIM to (1), we can construct the correction functional

$$
\begin{aligned}
& x_{n+1}(t)=x_{n}(t)+\frac{1}{\Gamma(\alpha)} \int_{0}^{t}(t-\xi)^{\alpha-1} \lambda(t, \xi) \\
& .\left[D_{*}^{\alpha} x_{n}(\xi)-\tilde{f}\left(x_{n}(\xi), x_{n}(\omega(\xi)),\right.\right. \\
& \left.\left.\int_{\xi-\tau}^{\xi} h\left(x_{n}(s), y_{n}(s)\right) d s, y_{n}(\xi), y_{n}(\phi(\xi))\right)\right] d \xi, \\
& 0=g\left(x_{n+1}(t), y_{n+1}(t)\right),
\end{aligned}
$$

where $\lambda(t, \xi)$ is a general Lagrange multiplier, which can be defined optimally by variational theory, and $\widetilde{f}$ denotes the restrictive variation; that is, $\delta \tilde{f}=0$. In order to obtain $\lambda(t, \xi)$, we select $\alpha=1$ (see [36]) and have

$$
\begin{aligned}
& \delta x_{n+1}(t)=\delta x_{n}(t)+\int_{0}^{t} \lambda(t, \xi) \delta\left[x_{n}^{\prime}(\xi)-\widetilde{f}\left(x_{n}(\xi),\right.\right. \\
& x_{n}(\omega(\xi)), \int_{\xi-\tau}^{\xi} h\left(x_{n}(s), y_{n}(s)\right) d s, y_{n}(\xi), \\
& \left.\left.y_{n}(\phi(\xi))\right)\right] d \xi .
\end{aligned}
$$

By using part integral to (4), the stationary conditions are obtained as

$$
\begin{array}{r}
\left.\frac{\partial \lambda(t, \xi)}{\partial \xi}\right|_{\xi=t}=0, \\
1+\left.\lambda(t, \xi)\right|_{\xi=t}=0 .
\end{array}
$$

Moreover, the general Lagrange multiplier can be readily identified by

$$
\lambda(t, \xi)=-1
$$

Therefore, the variational iteration formula can be written as

$$
\begin{aligned}
& x_{n+1}(t)=x_{n}(t)-J^{\alpha}\left[D_{*}^{\alpha} x_{n}(t)-f\left(x_{n}(t),\right.\right. \\
& x_{n}(\omega(t)), \int_{t-\tau}^{t} h\left(x_{n}(s), y_{n}(s)\right) d s, y_{n}(t), \\
& \left.\left.y_{n}(\phi(t))\right)\right] \\
& 0=g\left(x_{n+1}(t), y_{n+1}(t)\right) .
\end{aligned}
$$

Theorem 1. Let $x(t), x_{i}(t) \in\left(C^{1}[-\tau, T]\right)^{n_{1}}$ and $y(t), y_{i}(t) \in$ $\left(C^{1}[-\tau, T]\right)^{n_{2}}, i=1,2, \ldots$. Then the sequences $\left\{x_{n}(t)\right\}_{n=1}^{\infty}$ and $\left\{y_{n}(t)\right\}_{n=1}^{\infty}$ defined by (7a) and (7b) with $x_{0}(t)=\varphi(t), y_{0}(t)=$ $\psi(t)$, and $t \in[-\tau, 0]$ converge to the solutions of $(1)$.

Proof. From system (1), we have

$$
\begin{gathered}
x(t)=x(t)-J^{\alpha}\left[D_{*}^{\alpha} x(t)-f(x(t), x(\omega(t)),\right. \\
\left.\left.\int_{t-\tau}^{t} h(x(s), y(s)) d s, y(t), y(\phi(t))\right)\right], \\
0=g(x(t), y(t)) .
\end{gathered}
$$

Let $E_{n} x(t)=x_{n}(t)-x(t), E_{n} y(t)=y_{n}(t)-y(t), n=$ $0,1, \ldots$, and $E_{j} x(t)=E_{j} y(t)=0$ when $t<0, j=0,1, \ldots$

From (7a)-(8b), we obtain

$$
\begin{aligned}
& E_{n+1} x(t)=J^{\alpha}\left[f \left(x_{n}(t), x_{n}(\omega(t)),\right.\right. \\
& \quad \int_{t-\tau}^{t} h\left(x_{n}(s), y_{n}(s)\right) d s, y_{n}(t), y_{n}(\phi(t)) \\
& \quad-f\left(x(t), x(\omega(t)), \int_{t-\tau}^{t} h(x(s), y(s)) d s\right), \\
& y(t), y(\phi(t)))], \\
& 0=g\left(x_{n+1}(t), y_{n+1}(t)\right)-g(x(t), y(t)) .
\end{aligned}
$$

Based on the fact that the functions $f, g$ are smooth, the matrix $g_{y}$ is invertible, and hence we have

$$
\begin{aligned}
& E_{n+1} x(t)=J^{\alpha}\left[f_{1}^{\prime} E_{n} x(t)+f_{2}^{\prime} E_{n} x(\omega(t))\right. \\
& \quad+f_{3}^{\prime} \int_{t-\tau}^{t}\left[h\left(x_{n}(s), y_{n}(s)\right)-h(x(s), y(s))\right] d s \\
& \left.\quad+f_{4}^{\prime} E_{n} y(t)+f_{5}^{\prime} E_{n} y(\phi(t))\right], \\
& E_{n+1} y(t)=-g_{y}^{-1} g_{x} E_{n+1} x(t),
\end{aligned}
$$

where $f_{i}^{\prime}(i=1,2, \ldots, 5)$ denotes the partial derivative of the function $f$ to $i$ th variable. 
Let $l_{i}=\left\|f_{i}^{\prime}\right\|$ and $K=\left\|-g_{y}^{-1} g_{x}\right\|$. From (2), (10a), and (10b), we have

$$
\begin{aligned}
& \left(\begin{array}{l}
\left\|E_{n+1} x(t)\right\| \\
\left\|E_{n+1} y(t)\right\|
\end{array}\right) \\
& \quad \leq\left(\begin{array}{cc}
l_{1} & l_{4} \\
K l_{1} & K l_{4}
\end{array}\right)\left(\begin{array}{c}
J^{\alpha}\left\|E_{n} x(t)\right\| \\
J^{\alpha}\left\|E_{n} y(t)\right\|
\end{array}\right)
\end{aligned}
$$

$$
\begin{aligned}
& +\left(\begin{array}{cc}
l_{2} & l_{5} \\
K l_{2} & K l_{5}
\end{array}\right)\left(\begin{array}{c}
J^{\alpha}\left\|E_{n} x(\omega(t))\right\| \\
J^{\alpha}\left\|E_{n} y(\phi(t))\right\|
\end{array}\right) \\
& +l_{3} \tau\left(\begin{array}{cc}
\gamma_{1} & \gamma_{2} \\
K \gamma_{1} & K \gamma_{2}
\end{array}\right)\left(\begin{array}{c}
J^{\alpha} \max _{-\tau \leq \xi \leq T}\left\|E_{n} x(\xi)\right\| \\
J^{\alpha} \max _{-\tau \leq \xi \leq T}\left\|E_{n} y(\xi)\right\|
\end{array}\right) .
\end{aligned}
$$

We can derive

$$
\left(\begin{array}{l}
\left\|E_{n+1} x(t)\right\| \\
\left\|E_{n+1} y(t)\right\|
\end{array}\right) \leq\left(\begin{array}{cc}
l_{1}+l_{2}+l_{3} \tau \gamma_{1} & l_{4}+l_{5}+l_{3} \tau \gamma_{2} \\
K\left(l_{1}+l_{2}+l_{3} \tau \gamma_{1}\right) & K\left(l_{4}+l_{5}+l_{3} \tau \gamma_{2}\right)
\end{array}\right)\left(\begin{array}{c}
J^{\alpha} \max _{-\tau \leq t \leq T}\left\|E_{n} x(t)\right\| \\
J^{\alpha} \max _{-\tau \leq t \leq T}\left\|E_{n} y(t)\right\|
\end{array}\right) .
$$

Now, we proceed as follows:

$$
\begin{gathered}
\left(\begin{array}{c}
\left\|E_{1} x(t)\right\| \\
\left\|E_{1} y(t)\right\|
\end{array}\right) \leq\left(\begin{array}{cc}
l_{1}+l_{2}+l_{3} \tau \gamma_{1} & l_{4}+l_{5}+l_{3} \tau \gamma_{2} \\
K\left(l_{1}+l_{2}+l_{3} \tau \gamma_{1}\right) & K\left(l_{4}+l_{5}+l_{3} \tau \gamma_{2}\right)
\end{array}\right)\left(\begin{array}{cc}
\max _{-\tau \leq t \leq T} \frac{1}{\Gamma(\alpha+1)}\left\|E_{0} x(t)\right\| t^{\alpha} \\
\max _{-\tau \leq t \leq T} \frac{1}{\Gamma(\alpha+1)}\left\|E_{0} y(t)\right\| t^{\alpha}
\end{array}\right), \\
\left(\begin{array}{l}
\left\|E_{2} x(t)\right\| \\
\left\|E_{2} y(t)\right\|
\end{array}\right) \leq\left(\begin{array}{cc}
l_{1}+l_{2}+l_{3} \tau \gamma_{1} & l_{4}+l_{5}+l_{3} \tau \gamma_{2} \\
K\left(l_{1}+l_{2}+l_{3} \tau \gamma_{1}\right) & K\left(l_{4}+l_{5}+l_{3} \tau \gamma_{2}\right)
\end{array}\right)^{2}\left(\begin{array}{cc}
\max _{-\tau \leq t \leq T} \frac{1}{\Gamma(2 \alpha+1)}\left\|E_{0} x(t)\right\| t^{2 \alpha} \\
\max _{-\tau \leq t \leq T} \frac{1}{\Gamma(2 \alpha+1)}\left\|E_{0} y(t)\right\| t^{2 \alpha}
\end{array}\right), \\
\left(\begin{array}{l}
\left\|E_{3} x(t)\right\| \\
\left\|E_{3} y(t)\right\|
\end{array}\right) \leq\left(\begin{array}{cc}
l_{1}+l_{2}+l_{3} \tau \gamma_{1} & l_{4}+l_{5}+l_{3} \tau \gamma_{2} \\
K\left(l_{1}+l_{2}+l_{3} \tau \gamma_{1}\right) & K\left(l_{4}+l_{5}+l_{3} \tau \gamma_{2}\right)
\end{array}\right)^{3}\left(\begin{array}{cc}
\max _{-\tau \leq t \leq T} \frac{1}{\Gamma(3 \alpha+1)}\left\|E_{0} x(t)\right\| t^{3 \alpha} \\
\max _{-\tau \leq t \leq T} \frac{1}{\Gamma(3 \alpha+1)}\left\|E_{0} y(t)\right\| t^{3 \alpha}
\end{array}\right), \\
\left(\begin{array}{l}
\left\|E_{n} x(t)\right\| \\
\left\|E_{n} y(t)\right\|
\end{array}\right) \leq\left(\begin{array}{cc}
l_{1}+l_{2}+l_{3} \tau \gamma_{1} & l_{4}+l_{5}+l_{3} \tau \gamma_{2} \\
K\left(l_{1}+l_{2}+l_{3} \tau \gamma_{1}\right) & K\left(l_{4}+l_{5}+l_{3} \tau \gamma_{2}\right)
\end{array}\right)^{n}\left(\begin{array}{c}
\max _{-\tau \leq t \leq T} \frac{1}{\Gamma(n \alpha+1)}\left\|E_{0} x(t)\right\| t^{n \alpha} \\
\max _{-\tau \leq t \leq T} \frac{1}{\Gamma(n \alpha+1)}\left\|E_{0} y(t)\right\| t^{n \alpha}
\end{array}\right) .
\end{gathered}
$$

We have

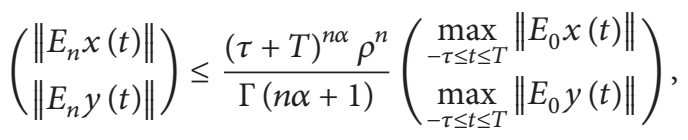

where $K, T, \tau, \gamma_{1}, \gamma_{2}, l_{i}(i=1,2, \ldots, 5), \max _{-\tau \leq t \leq T}\left\|E_{0} x(t)\right\|$, and $\max _{-\tau \leq t \leq T}\left\|E_{0} y(t)\right\|$ are constants and $\rho$ is the spectral radius of the iterative matrix in the above inequality.

We select $M=\lfloor n \alpha\rfloor$, and therefore $\Gamma(n \alpha+1) \geq \Gamma(M+1)$, $\Gamma(M+1)=M !$. Moreover, we have

$$
\left(\begin{array}{l}
\left\|E_{n} x(t)\right\| \\
\left\|E_{n} y(t)\right\|
\end{array}\right) \leq \frac{(\tau+T)^{n} \rho^{n}}{M !}\left(\begin{array}{c}
\max _{-\tau \leq t \leq T}\left\|E_{0} x(t)\right\| \\
\max _{-\tau \leq t \leq T}\left\|E_{0} y(t)\right\|
\end{array}\right) .
$$

By using Stirling's formula, we have

$$
\begin{aligned}
& \left(\begin{array}{l}
\left\|E_{n} x(t)\right\| \\
\left\|E_{n} y(t)\right\|
\end{array}\right) \\
& \leq \frac{[(\tau+T) \rho e / M]^{n}}{\sqrt{2 M \pi}(1+O(1 / M))}\left(\begin{array}{c}
\max _{-\tau \leq t \leq T}\left\|E_{0} x(t)\right\| \\
\max _{-\tau \leq t \leq T}\left\|E_{0} y(t)\right\|
\end{array}\right),
\end{aligned}
$$

and thus $\left(\left\|E_{n} x(t)\right\|,\left\|E_{n} y(t)\right\|\right)^{T} \rightarrow 0$ as $n \rightarrow \infty$.

If the right function $f\left(x(t), x(\omega(t)), \int_{t-\tau}^{t} h(x(s), y(s)) d s\right.$, $y(t), y(\phi(t)))=A x(t)+F\left(x(t), x(\omega(t)), \int_{t-\tau}^{t} h(x(s), y(s)) d s\right.$, 
$y(t), y(\phi(t)))$, we consider the initial value problems of fractional delay integrodifferential-algebraic equations

$$
\begin{aligned}
& D_{*}^{\alpha} x(t)=A x(t)+F(x(t), x(\omega(t)), \\
& \left.\quad \int_{t-\tau}^{t} h(x(s), y(s)) d s, y(t), y(\phi(t))\right), t \in[0, T], \\
& 0=g(x(t), y(t)), \quad t \in[0, T], \\
& x(t)=\varphi(t), \\
& y(t)=\psi(t),
\end{aligned}
$$$$
t \in[-\tau, 0],
$$

where the matrix $A=\operatorname{diag}\left(a_{11}, a_{22}, \ldots, a_{n_{1} n_{1}}\right) \in R^{n_{1} \times n_{1}}, F$ : $R^{n_{1}} \times R^{n_{1}} \times R^{n_{1}} \times R^{n_{2}} \times R^{n_{2}} \rightarrow R^{n_{1}}$, and $g: R^{n_{1}} \times R^{n_{2}} \rightarrow R^{n_{2}}$ are smooth vector functions on the real Euclidean spaces, and $h: R^{n_{1}} \times R^{n_{2}} \rightarrow R^{n_{1}}$ satisfies the Lipschitz condition

$$
\left\|h\left(v_{1}, \theta_{1}\right)-h\left(v_{2}, \theta_{2}\right)\right\| \leq \gamma_{1}\left\|v_{1}-v_{2}\right\|+\gamma_{2}\left\|\theta_{1}-\theta_{2}\right\|,
$$

where $v_{1}, v_{2}, \theta_{1}, \theta_{2}, \varphi, \psi, g_{y}, f_{i}^{\prime}$ are defined in the same way as those in system (1). We assume that system (17) has smooth solutions $x(t), y(t)$.

Applying the VIM to (17), we can construct the correction functionals

$$
\begin{aligned}
& x_{n+1}(t)=x_{n}(t)+\frac{1}{\Gamma(\alpha)} \int_{0}^{t}(t-\xi)^{\alpha-1} \Lambda(t, \xi) \\
& \cdot\left[D_{*}^{\alpha} x_{n}(\xi)-A x_{n}(\xi)-\widetilde{F}\left(x_{n}(\xi), x_{n}(\omega(\xi)),\right.\right. \\
& \left.\left.\quad \int_{\xi-\tau}^{\xi} h\left(x_{n}(s), y_{n}(s)\right) d s, y_{n}(\xi), y_{n}(\phi(\xi))\right)\right] d \xi, \\
& 0=g\left(x_{n+1}(t), y_{n+1}(t)\right) .
\end{aligned}
$$

We select $\alpha=1$, and thus

$$
\begin{aligned}
& \delta x_{n+1}(t)=\delta x_{n}(t)+\int_{0}^{t} \Lambda(t, \xi) \delta\left[x_{n}^{\prime}(\xi)-A x_{n}(\xi)\right. \\
& -\widetilde{F}\left(x_{n}(\xi), x_{n}(\omega(\xi)), \int_{\xi-\tau}^{\xi} h\left(x_{n}(s), y_{n}(s)\right) d s,\right. \\
& \left.\left.y_{n}(\xi), y_{n}(\phi(\xi))\right)\right] d \xi .
\end{aligned}
$$

By using part integral to (19a) and (19b), the stationary conditions are obtained as

$$
\begin{array}{r}
\left.\frac{\partial \Lambda(t, \xi)}{\partial \xi}\right|_{\xi=t}+A \Lambda(t, \xi)=0 \\
E+\left.\Lambda(t, \xi)\right|_{\xi=t}=0,
\end{array}
$$

and, moreover, the general Lagrange multiplier can be readily identified by

$$
\Lambda(t, \xi)=\left(-e^{a_{11}(t-\xi)},-e^{a_{22}(t-\xi)}, \ldots,-e^{a_{n_{1} n_{1}}(t-\xi)}\right)^{T} .
$$

Theorem 2. Let $x(t), x_{i}(t) \in\left(C^{1}[-\tau, T]\right)^{n_{1}}$ and $y(t), y_{i}(t) \in$ $\left(C^{1}[-\tau, T]\right)^{n_{2}}, i=1,2, \ldots$. Then the sequences $\left\{x_{n}(t)\right\}_{n=1}^{\infty}$ and $\left\{y_{n}(t)\right\}_{n=1}^{\infty}$ defined by (19a) and (19b) with $x_{0}(t)=\varphi(t), y_{0}(t)=$ $\psi(t)$, and $t \in[-\tau, 0]$ converge to the solutions of $(17)$.

Proof. The proof process is similar to that in system (1).

In general, the Lagrange multiplier $\Lambda(t, \xi)$ obtained with exponential form can increase convergence speed of iterative sequences.

\section{Special Cases}

Remark 3. If the right function of system (1) has no integral item and no delay item, it becomes the fractional differentialalgebraic equation. The results obtained are consistent with the ones discussed in [36]. Moreover, we present a new way to prove the convergence.

Remark 4. When $\alpha=1$, system (1) can be written as the delay integrodifferential-algebraic equation. Moreover, if the right function of system (1) has no integral item, it becomes the delay differential-algebraic equation discussed in [35].

In conclusion, we get the more general result, which extends the conclusions of existing literature.

\section{Illustrative Examples}

In this section, some illustrative examples are given to show the efficiency of the VIM for solving fractional delay differential-algebraic equations.

Example 1. Consider the initial value problem of fractional delay differential-algebraic equation

$$
\begin{aligned}
D_{*}^{\alpha} x(t) & =x\left(\frac{t}{2}\right) y(t)-\frac{t^{2}}{8} y(t)-\frac{t^{5}-t^{3}}{8}+\frac{6 t^{1.5}}{\Gamma(2.5)}, \\
t \in[0, T], & \\
0 & =x(t)-t y(t)+t^{2}, \quad t \in[0, T], \\
x(0) & =0, \\
y(0) & =0 .
\end{aligned}
$$

When $\alpha=1.5$, the exact solution of system (23) is

$$
\begin{aligned}
& x(t)=t^{3}, \\
& y(t)=t^{2}+t .
\end{aligned}
$$

Applying the VIM to (23), we can construct the correction functional

$$
\begin{aligned}
& x_{n+1}(t)=x_{n}(t)-J^{1.5}\left[D_{*}^{1.5} x_{n}(t)-x_{n}\left(\frac{t}{2}\right) y_{n}(t)\right. \\
& \left.+\frac{t^{2}}{8} y_{n}(t)+\frac{t^{5}-t^{3}}{8}-\frac{6 t^{1.5}}{\Gamma(2.5)}\right] \\
& y_{n+1}(t)=\frac{x_{n+1}(t)}{t}+t
\end{aligned}
$$


Moreover, the iteration sequence with the initial approximations $x_{0}(t)=0$ and $y_{0}(t)=0$ is obtained from (25a) and (25b) as follows:

$$
\begin{aligned}
x_{1}(t) & =0.9945231827 t^{3}+0.171607664 t^{3.5} \\
+ & 0.101083967 t^{4.5}, \\
y_{1}(t) & =t+0.9945231827 t^{2}+0.171607664 t^{2.5} \\
& +0.101083967 t^{3.5}, \\
x_{2}(t) & =0.9998023770 t^{3}+0.0066196316 t^{3.5} \\
- & 0.000964871 t^{4.5}+0.0098023770 t^{5} \\
- & 0.420964871 t^{6}, \\
y_{2}(t) & =t+0.9998023770 t^{2}+0.0066196316 t^{2.5} \\
- & 0.000964871 t^{3.5}+0.0098023770 t^{4} \\
- & 0.420964871 t^{5}, \\
x_{3}(t) & =0.9999891996 t^{3}+0.000403828233 t^{3.5} \\
- & 0.0000291441 t^{4.5}-0.11787329 t^{5} \\
+ & 0.003079509167 t^{6}+0.000009896323 t^{6.5} \\
+ & 0.000110548961 t^{7.5}, \\
y_{3}(t) & =t+0.9999891996 t^{2}+0.000403828233 t^{2.5} \\
- & 0.0000291441 t^{3.5}-0.11787329 t^{4} \\
+ & 0.003079509167 t^{5}+0.000009896323 t^{5.5} \\
\vdots & 0.000110548961 t^{6.5}, \\
&
\end{aligned}
$$

The approximate solution and exact solution are plotted in Figures 1 and 2. The imaginary line is the curve of the approximate solution, and the solid line is the curve of the exact solution, which shows that the method gives a very good approximation to the exact solution.

Example 2. Consider the initial value problem of fractional delay differential-algebraic equation

$$
\begin{aligned}
D_{*}^{\alpha} x(t) & =-x^{2}\left(\frac{t}{2}\right)+y(t)-\sin (t), \quad t \in[0, T], \\
0 & =x(t)-y(t)-e^{-t}+\sin (t), \quad t \in[0, T], \\
x(0) & =1, \\
y(0) & =0 .
\end{aligned}
$$

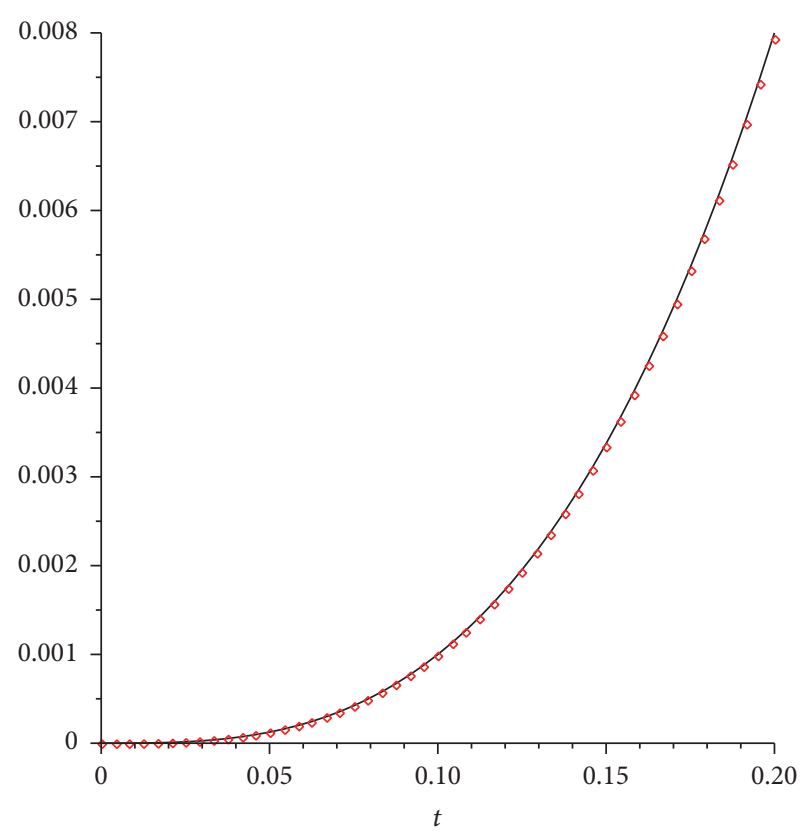

FIGURE 1: The comparison of the exact and approximate solutions of $x$.

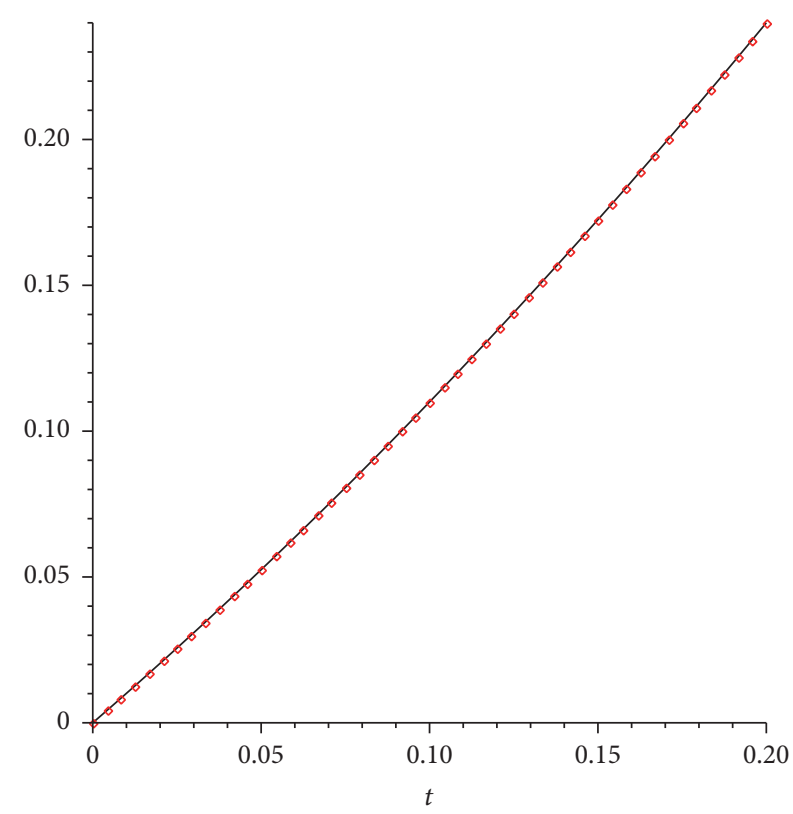

FIGURE 2: The comparison of the exact and approximate solutions of $y$.

When $\alpha=1$, the exact solution of system (27) is

$$
\begin{aligned}
& x(t)=e^{-t}, \\
& y(t)=\sin (t) .
\end{aligned}
$$


TABLE 1: The errors of the iteration for $x(t)$.

\begin{tabular}{lcccc}
\hline$t$ & $t=0.02$ & $t=0.06$ & $t=0.1$ & $t=0.3$ \\
\hline$x_{3}(t)$ & 0.009999833333 & 0.9982000000 & 0.9950000000 & 1.4843655750 \\
$x(t)$ & 0.009999833334 & 0.9982005399 & 0.9950041653 & 1.4843717880 \\
Error & $6.7000 E-09$ & $5.3990 E-07$ & $4.1656 E-06$ & $6.2130 E-06$ \\
\hline
\end{tabular}

TABLE 2: The errors of the iteration for $y(t)$.

\begin{tabular}{lcccc}
\hline$t$ & $t=0.02$ & $t=0.06$ & $t=0.1$ & $t=0.3$ \\
\hline$y_{3}(t)$ & 0.01999866670 & 0.05996399737 & 0.09983329948 & 0.2955202500 \\
$y(t)$ & 0.01999866666 & 0.05996400648 & 0.09983341666 & 0.2954917734 \\
Error & $4.1000 E-11$ & $9.1100 E-09$ & $1.1718 E-07$ & $2.8476 E-05$ \\
\hline
\end{tabular}

Using the VIM in the previous section, we construct the following correction functional:

$$
\begin{aligned}
& x_{n+1}(t) \\
& =x_{n}(t) \\
& -J^{1}\left[D_{*}^{1} x_{n}(t)+x_{n}^{2}\left(\frac{t}{2}\right)-y_{n}(t)+\sin (t)\right], \\
& 0=x_{n+1}(t)-y_{n+1}(t)-e^{-t}+\sin (t) \text {. }
\end{aligned}
$$

Moreover, the iteration sequence with the initial approximations $x_{0}(t)=1$ and $y_{0}(t)=0$ is obtained from (29a) and (29b) as follows:

$$
\begin{gathered}
x_{1}(t)=1-t+\frac{1}{2} t^{2}+\frac{1}{24} t^{4} \\
y_{1}(t)=t-\frac{1}{3} t^{3}+\frac{1}{720} t^{6} \\
x_{2}(t)=1-t+\frac{1}{2} t^{2}-\frac{1}{6} t^{3}-\frac{1}{96} t^{4}-\frac{1}{240} t^{5}+o\left(t^{5}\right), \\
y_{2}(t)=t-\frac{1}{3} t^{3}+\frac{5}{96} t^{4}+\frac{1}{240} t^{5}+o\left(t^{5}\right) \\
x_{3}(t)=1-t+\frac{1}{2} t^{2}-\frac{1}{6} t^{3}+\frac{1}{24} t^{4}-\frac{13}{3840} t^{5}+o\left(t^{5}\right), \\
y_{3}(t)=t-\frac{1}{6} t^{3}-\frac{13}{3840} t^{5}+o\left(t^{5}\right) \\
\vdots
\end{gathered}
$$

When the iteration number $n=3$, the corresponding relative errors are shown in Tables 1 and 2.

Example 3. Consider the initial value problem of a fractional delay integrodifferential-algebraic equation

$$
\begin{aligned}
D_{*}^{\alpha} x(t)= & 4 x\left(\frac{t}{2}\right) y(t)+y^{2}(t)-y^{3}(t) \\
& +\frac{2}{\Gamma(3-\alpha)} t^{2-\alpha}
\end{aligned}
$$

$$
\begin{aligned}
& +\int_{0}^{t}(3 x(s)-2 y(s)) d s, \quad t \in[0, T], \\
0 & =y^{2}(t)-x(t), \quad t \in[0, T], \\
x(0) & =0, \\
y(0)= & 0 .
\end{aligned}
$$

When $\alpha=1.8$, the exact solutions of system (31) are

$$
\begin{aligned}
& x(t)=t^{2}, \\
& y(t)=t .
\end{aligned}
$$

Using the VIM in the previous section, we construct the following correction functionals:

$$
\begin{aligned}
& x_{n+1}(t)=x_{n}(t)-J^{1.8}\left[D_{*}^{1.8} x_{n}(t)-4 x_{n}\left(\frac{t}{2}\right) y_{n}(t)\right. \\
& \quad-y_{n}^{2}(t) y_{n}^{3}(t)-\frac{2}{\Gamma(1.2)} t^{0.2} \\
& \left.\quad-\int_{0}^{t}\left(3 x_{n}(s)-2 y_{n}(s)\right) d s\right], \\
& 0=y_{n+1}^{2}(t)-x_{n+1}(t) .
\end{aligned}
$$

Moreover, the iteration sequence with the initial approximations $x_{0}(t)=t$ and $y_{0}(t)=t$ is obtained from (33a) and (33b) as follows:

$$
\begin{aligned}
& x_{1}(t)=1.040023124 t^{2}-0.8231479768 t^{4.8}, \\
& y_{1}(t)=1.040023124 t-0.8231479768 t^{3.8}, \\
& x_{2}(t)=0.9991113151 t^{2}+0.100545240 t^{3.8} \\
& \quad-0.0003301028 t^{4.8}-0.0008794265 t^{6.6}+o\left(t^{7}\right), \\
& y_{2}(t)=0.9991113151 t+0.100545240 t^{2.8} \\
& \quad-0.0003301028 t^{3.8}-0.0008794265 t^{5.6}+o\left(t^{6}\right) .
\end{aligned}
$$




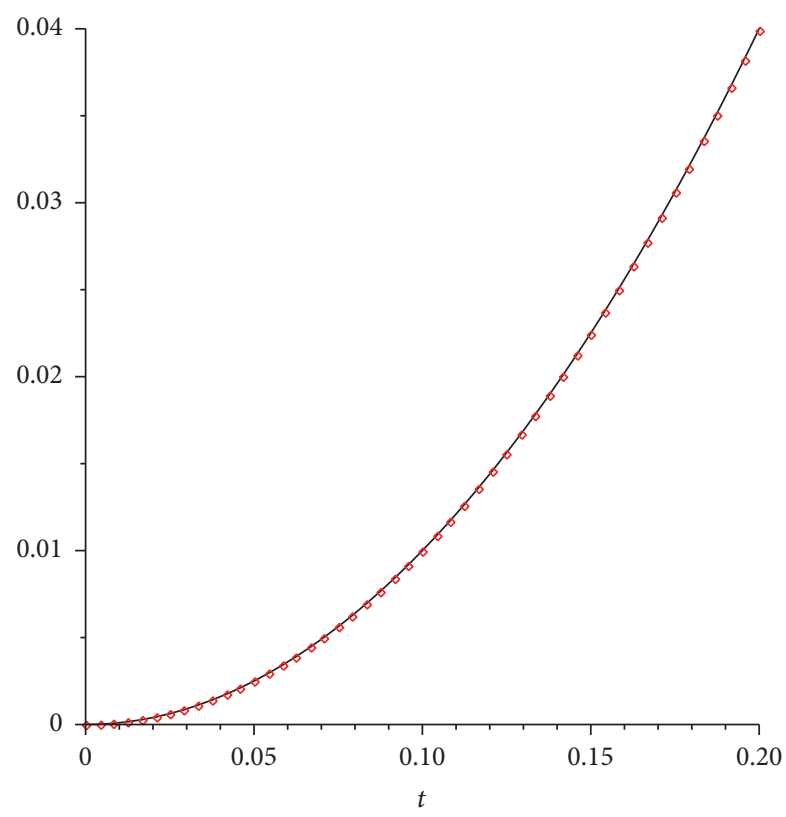

FIGURE 3: The comparison of the exact and approximate solutions of $x$

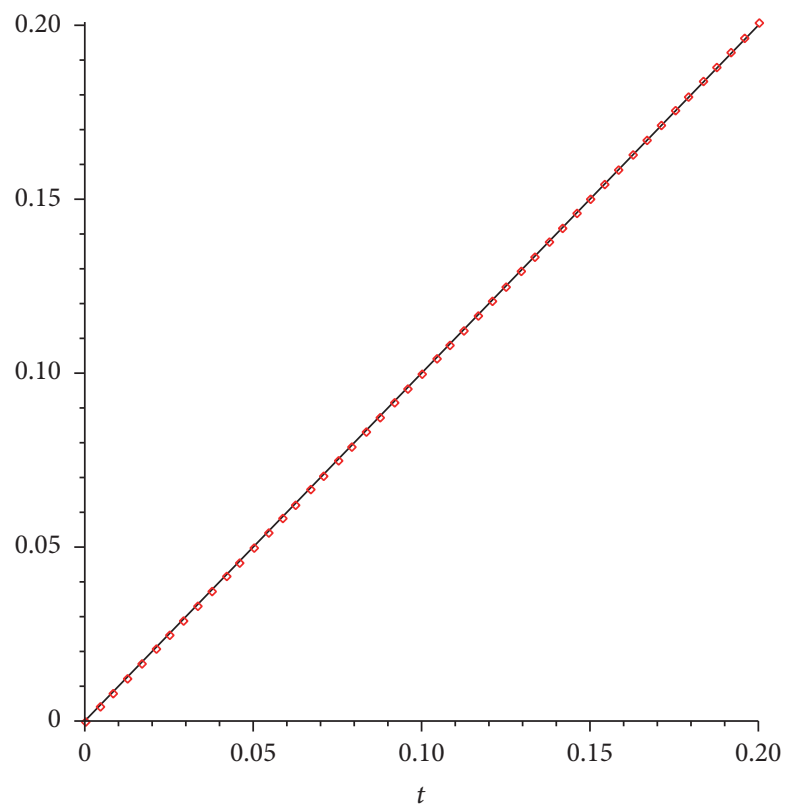

FIGURE 4: The comparison of the exact and approximate solutions of $y$.

The approximate solutions and exact solutions are plotted in Figures 3 and 4 . The imaginary line is the curve of the approximate solution, and the solid line is the curve of the exact solution, which shows that the method gives a very good approximation to the exact solution.
Example 4. Consider the initial value problem of a fractional delay integrodifferential-algebraic equation

$$
\begin{aligned}
D_{*}^{\alpha} x(t)= & x(t)-2 x\left(\frac{t}{2}\right) y\left(\frac{t}{4}\right) \\
& +\int_{0}^{t}[x(u)-\sqrt{y(u)}] d u, \quad t \in[0,1], \\
0 & =e^{-t} x(t)-y(t), \quad t \in[0,1] \\
x(0) & =1 \\
y(0) & =1 .
\end{aligned}
$$

When $\alpha=1$, the exact solution of system (35) is

$$
\begin{aligned}
& x(t)=e^{-t}, \\
& y(t)=e^{-2 t} .
\end{aligned}
$$

We select $\Lambda(t, s)=-e^{(t-s)}$, and, using the VIM in the previous section, we construct the following correction functionals:

$$
\begin{aligned}
& x_{n+1}(t)=x_{n}(t)-J^{1}\left[e ^ { t - s } \left(D_{*}^{1} x_{n}(t)-x_{n}(t)\right.\right. \\
& -2 x_{n}\left(\frac{t}{2}\right) y_{n}\left(\frac{t}{4}\right) \\
& \left.\left.+\int_{0}^{t}\left[x_{n}(u)-\sqrt{y_{n}(u)}\right] d u\right)\right], \\
& 0=e^{-t} x_{n+1}(t)-y_{n+1}(t) .
\end{aligned}
$$

Moreover, the iteration sequence with the initial approximations $x_{0}(t)=1$ and $y_{0}(t)=1$ is obtained from (37a) and (37b) as follows:

$$
\begin{gathered}
x_{1}(t)=1-t, \\
y_{1}(t)=1-2 t, \\
x_{2}(t)=1-t+\frac{1}{2} t^{2}, \\
y_{2}(t)=1-2 t+2 t^{2}, \\
x_{3}(t)=1-t+\frac{1}{2} t^{2}-\frac{7}{24} t^{3}, \\
y_{3}(t)=1-2 t+2 t^{2}-\frac{4}{3} t^{3},
\end{gathered}
$$

When the iteration number $n=3$, the corresponding relative errors are shown in Tables 3 and 4 .

To compare the convergence speed of iterative sequences with different Lagrange multiplier $\Lambda(t, s)$, then, we 
TABLE 3: The errors of the iteration for $x(t)$.

\begin{tabular}{lcccc}
\hline$t$ & $t=0.1$ & $t=0.15$ & $t=0.2$ & $t=0.3$ \\
\hline$x_{3}(t)$ & 0.9047083333 & 0.8602656250 & 0.8176666667 & 0.7371250000 \\
$x(t)$ & 0.9048374180 & 0.8607079764 & 0.8187307531 & 0.7408182207 \\
Error & $1.2908 E-04$ & $4.4235 E-04$ & $1.0640 E-03$ & $3.6932 E-03$ \\
\hline
\end{tabular}

TABLE 4: The errors of the iteration for $y(t)$.

\begin{tabular}{lcccc}
\hline$t$ & $t=0.1$ & $t=0.15$ & $t=0.2$ & $t=0.3$ \\
\hline$y_{3}(t)$ & 0.8186666667 & 0.7405000000 & 0.6693333333 & 0.5440000000 \\
$y(t)$ & 0.8187307531 & 0.7408182207 & 0.6703200460 & 0.5488116361 \\
Error & $6.4086 E-05$ & $3.1822 E-04$ & $9.8671 E-04$ & $4.8116 E-03$ \\
\hline
\end{tabular}

TABLE 5: The error comparison of $x(t)$ with different $\Lambda(t, s)$.

\begin{tabular}{lccccc}
\hline$t$ & $t=0.01$ & $t=0.03$ & $t=0.05$ & $t=0.07$ & $t=0.09$ \\
\hline Error $x_{1}$ & $1.2540 E-07$ & $3.4085 E-06$ & $1.5882 E-05$ & $4.3861 E-05$ & $9.3810 E-05$ \\
Error $x_{2}$ & $3.9850 E-02$ & $1.1866 E-01$ & $1.9627 E-01$ & $2.7266 E-01$ & $3.8518 E-01$ \\
\hline
\end{tabular}

TABLE 6: The error comparison of $y(t)$ with different $\lambda(t, s)$.

\begin{tabular}{lccccc}
\hline$t$ & $t=0.01$ & $t=0.03$ & $t=0.05$ & $t=0.07$ & $t=0.09$ \\
\hline Error $y_{1}$ & $6.6000 E-09$ & $5.3360 E-07$ & $4.0847 E-06$ & $1.5568 E-05$ & $4.2211 E-05$ \\
Error $y_{2}$ & $6.0052 E-02$ & $1.8051 E-01$ & $3.0149 E-01$ & $4.2307 E-01$ & $5.4544 E-01$ \\
\hline
\end{tabular}

select $\Lambda(t, s)=-1$ and construct the following correction functionals

$$
\begin{aligned}
& x_{n+1}(t)=x_{n}(t)-J^{1}\left[D_{*}^{1} x_{n}(t)-x_{n}(t)\right. \\
& \left.\quad-2 x_{n}\left(\frac{\mathrm{t}}{2}\right) y_{n}\left(\frac{t}{4}\right)+\int_{0}^{t}\left[x_{n}(u)-\sqrt{y_{n}(u)}\right] d u\right], \\
& 0=e^{-t} x_{n+1}(t)-y_{n+1}(t) .
\end{aligned}
$$

Moreover, the iteration sequence with the initial approximations $x_{0}(t)=1$ and $y_{0}(t)=1$ is obtained from (39a) and (39b), and when the iteration number $n=3$, the error comparisons of different $\Lambda(t, s)$ are shown in Tables 5 and 6.

The Error $x_{1}$ denotes the error of $x(t)$ with $\Lambda(t, s)=-e^{t-s}$, and the Error $x_{2}$ denotes the error of $x(t)$ with $\Lambda(t, s)=-1$.

The Error $y_{1}$ denotes the error of $y(t)$ with $\Lambda(t, s)=-e^{t-s}$, and the Error $y_{2}$ denotes the error of $y(t)$ with $\Lambda(t, s)=-1$.

Tables 5 and 6 show that the Lagrange multiplier $\Lambda(t, \xi)$ with exponential form can increase convergence speed of iterative sequences in Example 4.

In conclusion, the convergence speed of iterative sequences can be increased by selecting the appropriate restrictive variation and constructing the corresponding Lagrange multiplier. The construction techniques of different Lagrange multipliers are discussed in [24].

\section{Conclusion}

In this paper, the convergence of VIM for FDIDAEs is established. Theoretical analysis and numerical experiments show that VIM can be used efficiently to solve FDIDAEs. This method offers significant advantages in terms of applicability and computational efficacy and accuracy.

\section{Competing Interests}

The authors declare that they have no competing interests.

\section{Acknowledgments}

The authors would like to thank the Scientific Research Project of Hunan Provincial Education Department (no. 17B258) and the projects from NSF of China (nos. 11301448 and 11671343).

\section{References}

[1] N. M. Chuong, T. D. Ke, and N. N. Quan, "Stability for a class of fractional partial integro-differential equations," Journal of Integral Equations \& Applications, vol. 26, no. 2, pp. 145-170, 2014.

[2] S. Mathews, A. Reid, C. L. Tian, and Q. Cai, "Lyapunov stability solutions of fractional integro-differential equations," International Journal of Mathematics \& Mathematical Sciences, vol. 47, no. 45-48, pp. 2503-2507, 2004. 
[3] Z. M. Mao and W. H. Yuan, "Asymptotic stability of fractional impulsive neutral stochastic partial integro-differential equations with state-dependent delay," Electronic Journal of Differential Equations, vol. 2013, no. 206, pp. 1-29, 2013.

[4] A. Arikoglu and I. Ozkol, "Solution of fractional integrodifferential equations by using fractional differential transform method," Chaos, Solitons \& Fractals, vol. 40, no. 2, pp. 521-529, 2009.

[5] X. Ma and C. Huang, "Spectral collocation method for linear fractional integro-differential equations," Applied Mathematical Modelling, vol. 38, no. 4, pp. 1434-1448, 2014.

[6] X. Ma and C. Huang, "Numerical solution of fractional integrodifferential equations by a hybrid collocation method," Applied Mathematics \& Computation, vol. 219, no. 12, pp. 6750-6760, 2013.

[7] E. A. Rawashdeh, "Numerical solution of fractional integrodifferential equations by collocation method," Applied Mathematics \& Computation, vol. 176, no. 1, pp. 1-6, 2006.

[8] Y. Nawaz, "Variational iteration method and homotopy perturbation method for fourth-order fractional integro-differential equations," Computers and Mathematics with Applications, vol. 61, no. 8, pp. 2330-2341, 2011.

[9] M. H. Saleh, D. S. Mohamed, M. H. Ahmed, and M. K. Marjan, "System of linear fractional integro-differential equations by using Adomian decomposition method," International Journal of Computer Applications, vol. 121, no. 24, pp. 9-19, 2015.

[10] L. Huang, X.-F. Li, Y. Zhao, and X.-Y. Duan, "Approximate solution of fractional integro-differential equations by Taylor expansion method," Computers \& Mathematics with Applications, vol. 62, no. 3, pp. 1127-1134, 2011.

[11] S. L. Campbell and V. H. Linh, "Stability criteria for differentialalgebraic equations with multiple delays and their numerical solutions," Applied Mathematics \& Computation, vol. 208, no. 2, pp. 397-415, 2009.

[12] R. Miyazaki, "Asymptotic behavior for linear delay-differential equations with periodically oscillatory coefficients," Journal of Mathematical Analysis and Applications, vol. 204, no. 1, pp. 183205, 1996.

[13] F. Y. Xiao and C. J. Zhang, "Convergence of one-leg methods for a class of variable retarded differential algebraic equations," Journal on Numerical Methods and Computer Applications, vol. 29, no. 3, pp. 217-225, 2008.

[14] R. Hauber, "Numerical treatment of retarded differentialalgebraic equations by collocation methods," Advances in Computational Mathematics, vol. 7, no. 4, pp. 573-592, 1997.

[15] H. L. Liu and A. G. Xiao, "Convergence of linear multistep methods and one-leg methods for index-2 differential-algebraic equations with a variable delay," Advances in Applied Mathematics \& Mechanics, vol. 4, no. 5, pp. 636-646, 2012.

[16] H. L. Liu and A. G. Xiao, "Convergence of backward differentiation formulas for index-2 differential-algebraic equations with variable delay," Chinese Journal of Engineering Mathematics, vol. 28, no. 3, pp. 335-342, 2011.

[17] Y. Li, L. Sun, and Q. Yu, "Stability of two-step Runge-Kutta methods for neutral delay differential-algebraic equations," International Journal of Computer Mathematics, vol. 88, no. 2, pp. 375-383, 2011.

[18] J. J. Zhao, Y. Xu, S. Y. Dong, and M. Z. Liu, "Stability of the Rosenbrock methods for the neutral delay differential-algebraic equations," Applied Mathematics and Computation, vol. 168, no. 2, pp. 1128-1144, 2005.
[19] J.-P. Kauthen, "Implicit Runge-Kutta methods for some integrodifferential-algebraic equations," Applied Numerical Mathematics, vol. 13, no. 1-3, pp. 125-134, 1993.

[20] H. Yuan and J. Shen, "The stability of two-step Runge-Kutta methods for neutral delay integro differential-algebraic equations with many delays," Mathematical Problems in Engineering, vol. 2014, Article ID 918371, 7 pages, 2014.

[21] Y. Xu and J. Zhao, "Stability of Runge-Kutta methods for neutral delay-integro-differential-algebraic system," Mathematics and Computers in Simulation, vol. 79, no. 3, pp. 571-583, 2008.

[22] J. He, "A new approach to nonlinear partial differential equations," Communications in Nonlinear Science \& Numerical Simulation, vol. 2, no. 4, pp. 230-235, 1997.

[23] J.-H. He and X.-H. Wu, "Construction of solitary solution and compacton-like solution by variational iteration method," Chaos, Solitons and Fractals, vol. 29, no. 1, pp. 108-113, 2006.

[24] J.-H. He, "Variational iteration method-Some recent results and new interpretations," Journal of Computational and Applied Mathematics, vol. 207, no. 1, pp. 3-17, 2007.

[25] G.-Y. Wang, J.-H. He, and L.-F. Mo, "Variational iteration method for nonlinear oscillators: a comment on 'Application of laplace iteration method to study of nonlinear vibration of laminated composite plates,' Latin American Journal of Solids and Structures, vol. 11, no. 2, pp. 344-347, 2014.

[26] Y. Zhao and A. Xiao, "Variational iteration method for singular perturbation initial value problems," Computer Physics Communications, vol. 181, no. 5, pp. 947-956, 2010.

[27] Y. Zhao, A. Xiao, L. Li, and C. Zhang, "Variational iteration method for singular perturbation initial value problems with delays," Mathematical Problems in Engineering, vol. 2014, Article ID 850343, 8 pages, 2014.

[28] R. N. Prajapati, R. Mohan, and P. Kumar, "Numerical solution of generalized Abel's integral equation by variational iteration method," American Journal of Computational Mathematics, vol. 2, no. 4, pp. 312-315, 2012.

[29] H. Liu, A. Xiao, and L. Su, "Convergence of variational iteration method for second-order delay differential equations," Journal of Applied Mathematics, vol. 2013, Article ID 634670, 9 pages, 2013.

[30] X. Chen and L. Wang, "The variational iteration method for solving a neutral functional-differential equation with proportional delays," Computers and Mathematics with Applications, vol. 59, no. 8, pp. 2696-2702, 2010.

[31] G.-C. Wu, "A fractional variational iteration method for solving fractional nonlinear differential equations," Computers \& Mathematics with Applications, vol. 61, no. 8, pp. 2186-2190, 2011.

[32] H. Jafari and H. Tajadodi, "He's variational iteration method for solving fractional Riccati differential equation," International Journal of Differential Equations, vol. 2010, Article ID 764738, 8 pages, 2010.

[33] A.-M. Yang, J. Li, H. M. Srivastava, G.-N. Xie, and X.-J. Yang, "Local fractional laplace variational iteration method for solving linear partial differential equations with local fractional derivative," Discrete Dynamics in Nature and Society, vol. 2014, Article ID 365981, 8 pages, 2014.

[34] S. M. Amer, M. H. Saleh, M.-A. Mohamed, and N. S. Abdelrhman, "Variational iteration method and Adomian decomposition method for fourth-order fractional integro-differential equations," International Journal of Computer Applications, vol. 80, no. 6, pp. 7-14, 2013. 
[35] H. Liu, A. Xiao, and Y. Zhao, "Variational iteration method for delay differential-algebraic equations," Mathematical and Computational Applications, vol. 15, no. 5, pp. 834-839, 2010.

[36] B. Ibiş and M. Bayram, "Numerical comparison of methods for solving fractional differential-algebraic equations (FDAEs)," Computers \& Mathematics with Applications, vol. 62, no. 8, pp. 3270-3278, 2011. 


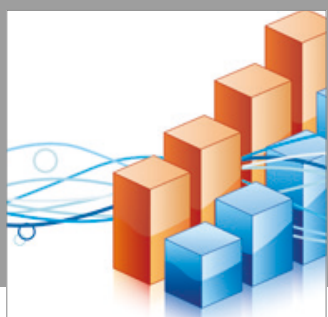

Advances in

Operations Research

vatersals

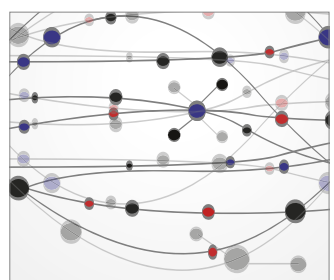

\section{The Scientific} World Journal
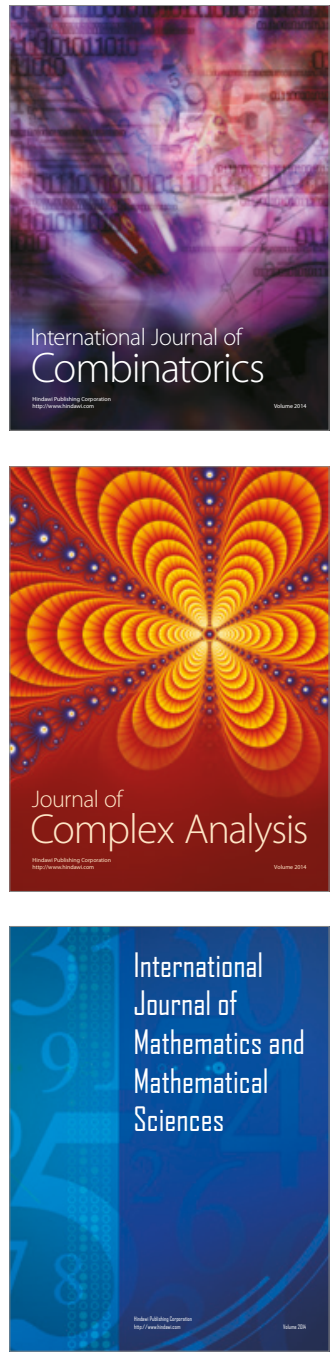
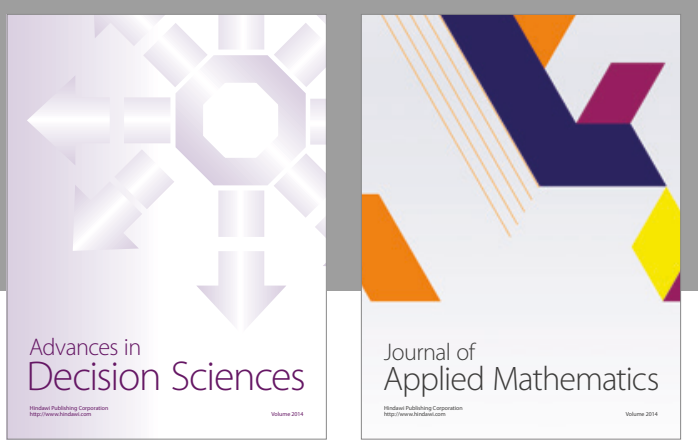

Algebra

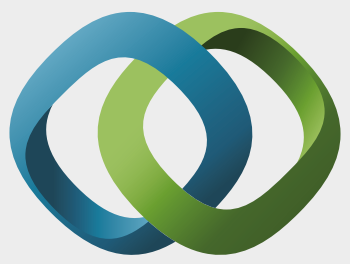

\section{Hindawi}

Submit your manuscripts at

https://www.hindawi.com
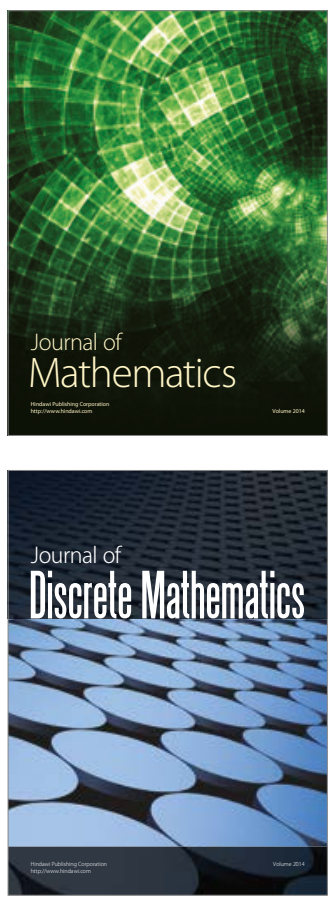

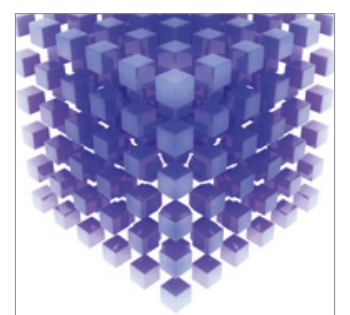

Mathematical Problems in Engineering
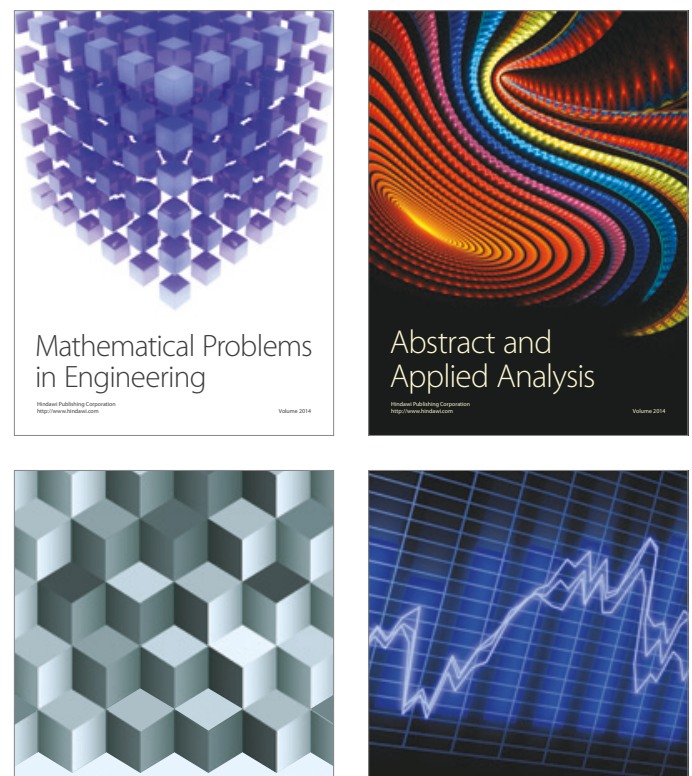

Journal of

Function Spaces

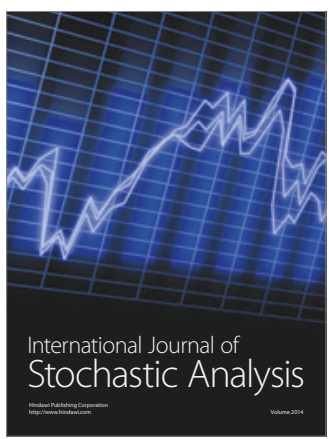

Probability and Statistics
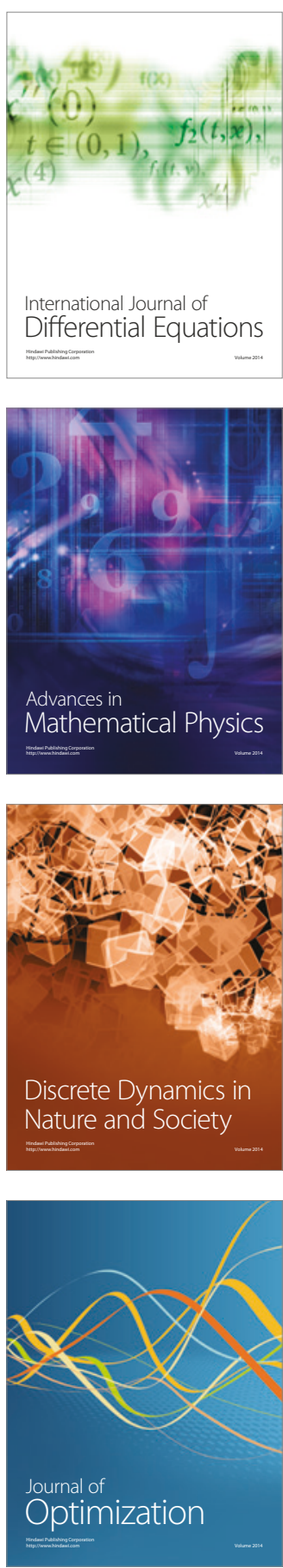